\title{
Associated production of a $W$ or $Z$ boson with bottom quarks at the Tevatron and the LHC
}

\author{
Fernando Febres Cordero \\ Universidad Simón Bolívar, Departamento de Física, Apartado 89000, Caracas 1080A, \\ Venezuela \\ E-mail: ffebres@usb.ve
}

\author{
Laura Reina \\ Florida State University, Physics Department, Tallahassee, FL 32306-4350, USA \\ E-mail: reina@hep.fsu.edu
}

\section{Doreen Wackeroth ${ }^{* *}$}

University at Buffalo, The State University of New York, Department of Physics, Buffalo, NY 14260-1500, USA

E-mail: dow@ubpheno.physics.buffalo.edu

\begin{abstract}
We present total and differential cross sections for $W b \bar{b}$ and $Z b \bar{b}$ production at the CERN Large Hadron Collider including Next-to-Leading Order (NLO) QCD corrections and full bottom-quark mass effects. We discuss the scale uncertainty of the total cross sections due to the residual renormalization- and factorization-scale dependence of the truncated perturbative series. We also discuss $b$-quark mass effects in kinematic distributions by comparing with a calculation that considers massless bottom quarks, as implemented in the Monte Carlo program MCFM. The effects of a non-zero bottom-quark mass $\left(m_{b}\right)$ cannot be neglected in phase-space regions where the relevant kinematic observable, such as the transverse momentum of the bottom quarks or the invariant mass of the bottom-quark pair, are of the order of $m_{b}$. Finally, we present the result of a detailed comparison of NLO QCD predictions for $W+b$-jet production with one or two jets with Tevatron data.
\end{abstract}

RADCOR 2009 - 9th International Symposium on Radiative Corrections (Applications of Quantum Field Theory to Phenomenology),

October 25 - 302009

Ascona, Switzerland

\footnotetext{
* Speaker.

${ }^{\dagger}$ On sabbatical leave at the Karlsruhe Institute of Technology (KIT).
} 


\section{Introduction}

The associated production of a weak gauge boson with one or two $b$ jets constitutes not only an important background process to Higgs boson searches for light Higgs bosons $\left(M_{H} \lesssim 135 \mathrm{GeV}\right)$, to single top-quark production and many searches for signals of new physics, but also represents a unique opportunity to test and improve the theoretical prediction for heavy quark jets at hadron colliders. The cross sections for $W$ and $Z$ boson production with $b$ jets has been measured at the Tevatron $p \bar{p}$ collider at Fermilab $(\sqrt{s}=1.96 \mathrm{TeV})$ by both the CDF [1,2] and D0 [3] collaborations, and these measurements will continue to improve once more data have been analyzed. Studying the same cross sections in the very different kinematic regimes available at the LHC $p p$ collider will then be of great interest and will represent a crucial test of our understanding of QCD at high-energy colliders.

The production of a $W$ or a $Z$ boson with up to two jets, one of which is a $b$ jet, has been calculated including NLO QCD corrections in the variable-flavor scheme (VFS) or five-flavornumber scheme (5FNS) [4], while the production of a $W$ or $Z$ boson with two $b$ jets has been derived at NLO in QCD using the fixed-flavor scheme (FFS) or four-flavor-number scheme (4FNS), first in the massless $b$-quark approximation $[5,6]$ and more recently including full $b$-quark mass effects $[7,8,9,10]$. In the FFS or 4FNS only massless-quark densities are considered in the initial state, while in a VFS or 5FNS an initial-state $b$-quark density is introduced. The two schemes amount to a different ordering of the perturbative series for the production cross section: in the 4FNS the perturbative series is ordered strictly by powers of the strong coupling $\alpha_{s}$, whereas in the 5FNS the introduction of a $b$-quark parton distribution function (PDF) allows to resum terms of the form $\alpha_{s}^{n} \ln \left(m_{b}^{2} / M^{2}\right)^{m}$ at all orders in $\alpha_{s}$ (for fixed order of logarithms $m$ ), where $M$ represents the upper integration limit of the $b$-quark transverse momentum and can be thought to be of the order of $M_{W}$ or $M_{Z}$. While the two approaches can give very different results at leading order (LO) in QCD, starting at NLO in QCD the total cross sections have been shown to be consistent within their respective theoretical uncertainties for both $H+1 b$-jet production [11] (for a brief review see also Ref. [12]) and single-top production [13]. In kinematical distributions, however, $b$-quark mass effects which are fully taken into account in the 4FNS can have a significant impact, particular in phase space regions where the relevant kinematic observable is of the order of $m_{b}$. Bottom-quark mass effects are also important in $W+1 b$-jet production and improved predictions at NLO QCD are provided in [14], where the calculations of $W b \bar{b}$ production in the 4FNS with massive $b$ quarks and $W b j$ production in the 5FNS have been merged. A similar study is currently in progress for $Z+1 b$-jet production [15]. Improving the predictions for $Z+1 b$-jet production will be particularly relevant at the LHC, where this process allows for a direct determination of the $b$-quark PDF, to be used in the prediction of $H+1 b$-jet production, a discovery channel for beyond-the-SM Higgs bosons with enhanced $b$-quark Yukawa couplings.

In these proceedings, we present results for $W b \bar{b}$ and $Z b \bar{b}$ production at the LHC, i.e. with both $b$ jets tagged in the final state, keeping the $W$ and $Z$ boson on shell. We discuss the impact of the $b$-quark mass on total kinematic distributions by comparing with a calculation which neglects the $b$-quark mass at NLO in QCD, as implemented in the MCFM package [16]. Finally, we present the results of a detailed comparison of NLO QCD predictions for $W+b$-jet production with one or two jets with Tevatron data. 


\section{2. $W b \bar{b}$ and $Z b \bar{b}$ production at NLO QCD}

At tree level, the production of a $W$ boson with a pair of bottom quarks consists of just one process, $q \bar{q}^{\prime} \rightarrow W b \bar{b}$, while $Z b \bar{b}$ production consists of two channels, namely $q \bar{q} \rightarrow Z b \bar{b}$ and $g g \rightarrow$ $Z b \bar{b}$. In order to compute these processes at NLO in QCD one needs to include one-loop virtual corrections to the tree-level processes, as well as all real radiation corrections with up to one extra parton in the final state, i. e. $q \bar{q}^{\prime} \rightarrow W b \bar{b}+g, g g, q \bar{q} \rightarrow Z b \bar{b}+g$, and the $q g$-initiated processes, $q g(\bar{q} g) \rightarrow W b \bar{b}+q^{\prime}\left(\bar{q}^{\prime}\right)$ and $q g(\bar{q} g) \rightarrow Z b \bar{b}+q(\bar{q})$. Details of the calculations and numerical results are provided in Refs. [7, 8, 9, 10].

The LO results are based on the one-loop evolution of $\alpha_{s}$ and the CTEQ6L1 set of PDFs [17], with $\alpha_{s}^{L O}\left(M_{Z}\right)=0.130$, while the NLO results use the two-loop evolution of $\alpha_{s}$ and the CTEQ6M set of PDFs, with $\alpha_{s}^{N L O}\left(M_{Z}\right)=0.118$. We use the $k_{T}$ jet algorithm with a pseudo-cone size of $R=0.7$ and we checked that our implementation of the $k_{T}$ jet algorithm coincides with the one in MCFM. We require all events to have a $b$-jet pair in the final state, with a transverse momentum either larger than $15 \mathrm{GeV}$ or $25 \mathrm{GeV}$ and require the pseudorapidities of both $b$ jets satisfy $\left|\eta^{b, \bar{b}}\right|<$ 2.5. We impose the same $p_{T}$ and $|\eta|$ cuts also on the extra jet that may arise due to hard noncollinear real emission of a parton, i. e. in the processes $W / Z b \bar{b}+g$ or $W / Z b \bar{b}+q(\bar{q})$. This hard non-collinear extra parton is treated either inclusively or exclusively. In the inclusive case we include both two- and three-jet events, while in the exclusive case we require exactly two jets in the event. Two-jet events consist of a $b$-jet pair that may also include a final-state light parton (gluon or quark) due to the applied recombination procedure. On the other hand, three-jet events consist of events containing a $b$-jet pair plus an extra light jet. We notice that, at NLO in QCD, all jets in three-jet events consist of a single parton.

In Fig. $1\left(W^{ \pm} b \bar{b}\right)$ and Fig. $2(Z b \bar{b})$ we illustrate the renormalization- and factorization-scale dependence of the LO and NLO total cross sections obtained for a massive $b$ quark, when $\mu=$ $\mu_{r}=\mu_{f}$ is varied between $\mu_{0} / 2$ and $2 \mu_{0}$, with $\mu_{0}=M_{V}+2 m_{b}(V=W, Z)$. We immediately notice that the impact of NLO QCD corrections is very large, in particular in the inclusive case in $W b \bar{b}$ production, where they increase the LO cross section by a factor between two and three depending on the scale. We also notice that the scale dependence of the NLO $W b \bar{b}$ cross section is worse than (inclusive case) or comparable to (exclusive case) the scale dependence of the LO cross section. This is different from what has been observed for the Tevatron [7, 9], and was first pointed out in a calculation with massless bottom quarks [6]. It is just a reminder of the fact that, at a given perturbative order, the uncertainty due to the residual renormalization- and factorization-scale dependence may underestimate the theoretical uncertainty due to missing higher-order corrections. A realistic determination of this uncertainty is usually much more complex and requires a thorough understanding of the perturbative structure of the cross section, in particular at the lowest orders of the perturbative expansion. In both $W b \bar{b}$ and $Z b \bar{b}$ production the NLO QCD corrections introduce a new production channel not present at LO, the $q g$-initiated processes, and as such introduces a LO scale dependence. The NLO $W b \bar{b}$ total cross section is particularly affected by this process because there is no $g g$-initiated process at LO. In $Z b \bar{b}$ production, however, this process is not dominant at NLO and its impact is therefore less pronounced. Indeed, the scale dependence of the exclusive $Z b \bar{b}$ cross section actually greatly improves at NLO in QCD, while the scale dependence of the inclusive one is only mildly better than at $\mathrm{LO}$, but not worse as it is the case in $W b \bar{b}$ production. 

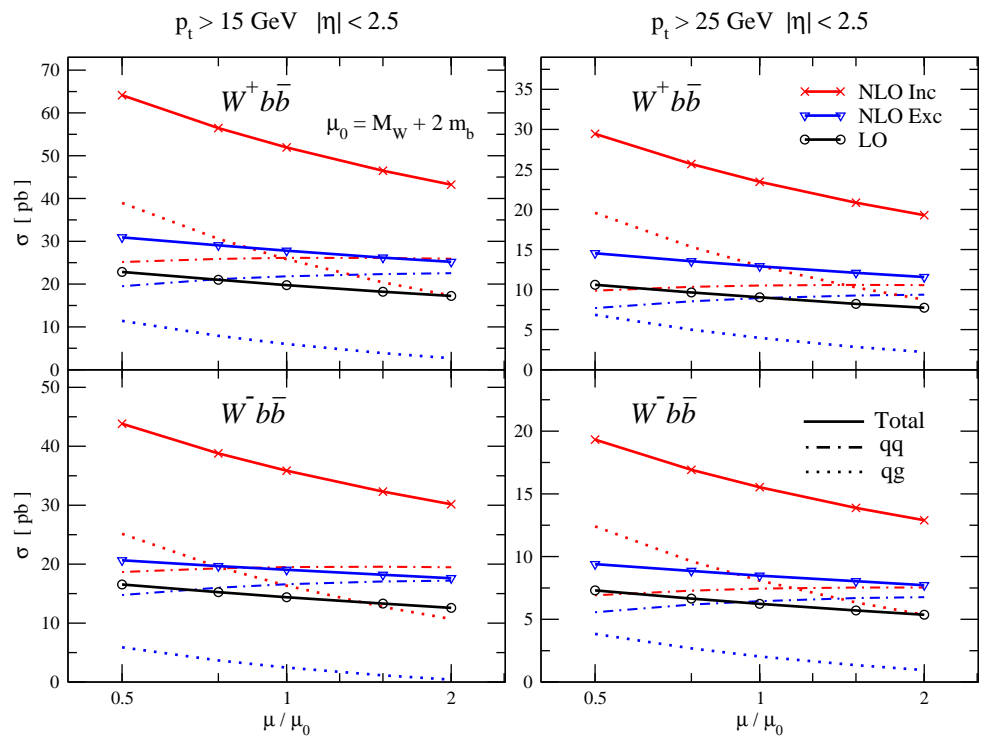

Figure 1: Dependence of the LO (black, solid), NLO exclusive (blue, solid), and NLO inclusive (red, solid) total cross sections for $W^{+} b \bar{b}$ and $W^{-} b \bar{b}$ production on the renormalization/factorization scales at the LHC $(\sqrt{s}=14 \mathrm{TeV})$, including full $b$-quark mass effects, when $\mu=\mu_{r}=\mu_{f}$ is varied between $\mu_{0} / 2$ and $2 \mu_{0}$ (with $\mu_{0}=M_{W}+2 m_{b}$ ). We also show the individual parton-level channels, $q \bar{q}^{\prime}$ (dash-dotted) and $q g+\bar{q} g$ (dotted), for the inclusive (red) and exclusive (blue) cases. Taken from Ref. [10].

Finally, the impact of the $q g$-initiated processes on the scale dependence of the total cross sections is larger in the inclusive than in the exclusive case because the exclusive cross section by definition discriminates against processes with more than two jets in the final state.

In Fig. 3, we illustrate $b$-quark mass effects on the example of $m_{b \bar{b}}$ distributions in $W^{-} b \bar{b}$ and $Z b \bar{b}$ production. These effects can impact the shape of the kinematic distributions in particular in phase space regions where the relevant kinematic observable is of the order of $m_{b}$. These effects can be approximated by rescaling the NLO cross section for $m_{b}=0$ with the ratio of LO cross sections for massive and massless bottom quarks as discussed in detail in [7, 8]. The total production cross sections are reduced by $b$-quark mass effects, and the effect is more pronounced the smaller the applied $p_{T}^{b}$ cut. However, these effects are in most cases smaller than the residual scale dependence at NLO in QCD, especially in $W b \bar{b}$ production for the inclusive case.

\section{Comparison of $W+b$-jet predictions with Tevatron data}

A recent measurement of the cross section of $W$ boson production in association with one or two $b$ jets by the CDF collaboration at the Tevatron finds [2]

$$
\sigma_{b-\text { jets }} \times \mathscr{B}(W \rightarrow \ell v)(\mathrm{CDF})=2.74 \pm 0.27 \text { (stat.) } \pm 0.42 \text { (syst.) pb }
$$

This $b$-jet cross section includes $W+2 b$-jets and $W+1 b$-jet contributions, and the NLO QCD predictions for both signatures have to be considered. The NLO QCD prediction for $W+2 b$-jets production is based on Refs. [7, 9] and the one for $W+1 b$-jet production on Ref. [14], where in 

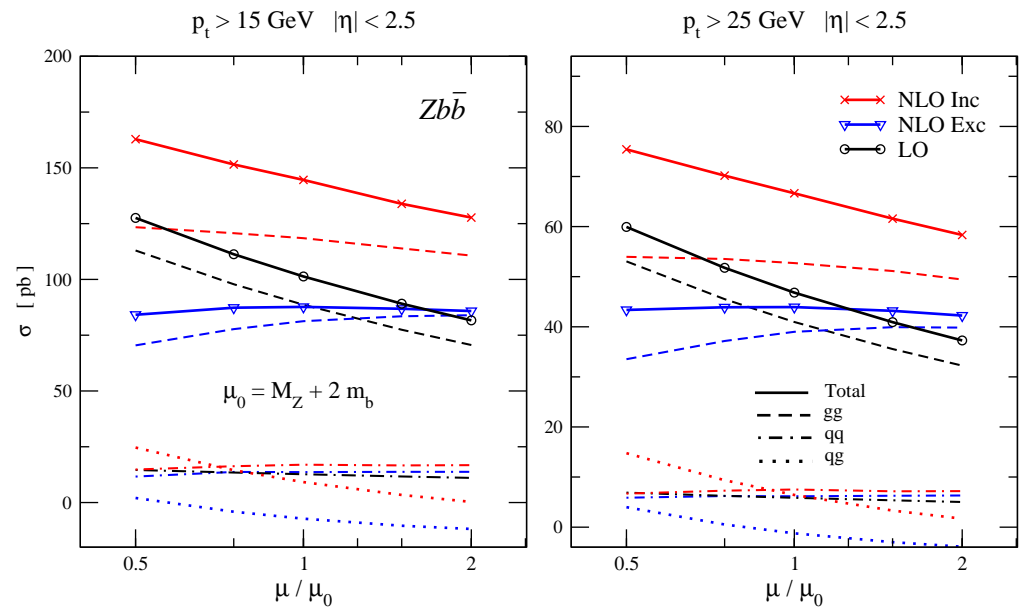

Figure 2: Dependence of the LO (black, solid), NLO exclusive (blue, solid), and NLO inclusive (red, solid) total cross sections for $Z b \bar{b}$ production on the renormalization/factorization scales at the $\operatorname{LHC}(\sqrt{s}=$ $14 \mathrm{TeV}$ ), including full $b$-quark mass effects, when $\mu=\mu_{r}=\mu_{f}$ is varied between $\mu_{0} / 2$ and $2 \mu_{0}$ (with $\mu_{0}=M_{Z}+2 m_{b}$ ). We also show the individual parton-level channels, $q \bar{q}^{\prime}$ (dash-dotted), $q g+\bar{q} g$ (dotted) and $g g$ (dashed), for LO and NLO (inclusive (red) and exclusive (blue)). Taken from Ref. [10].

both cases events with a non- $b$ jet that result in a three-jet event are discarded. In Ref. [14], the calculations of $W b \bar{b}$ production in the 4FNS and $W b j$ production in the 5FNS have been merged by consistently treating contributions which arise in both processes. In this way, the $b$-quark mass is taken into account, which cannot be neglected when one $b$ quark is treated fully inclusively, the tree-level $q g$-initiated process discussed earlier is improved by including NLO QCD corrections in the 5FNS, and large initial-state logarithms are resummed via the $b$-quark PDF approach. As a result of this improved treatment, theoretically more stable predictions for $W+1 b$-jet production are now available. Finally, combining the results of predictions for $W+2 b$-jets and $W+1 b$-jet production, thereby doubling the weights of $2 b$-jet events to obtain jet-level cross sections, yields the following NLO QCD prediction (with $\mu_{r}=\mu_{f}=M_{W}$ for the central value) [18]:

$$
\sigma_{b-\text { jets }} \times \mathscr{B}(W \rightarrow \ell v)(\mathrm{NLO} \mathrm{QCD})=1.22 \pm 0.14 \text { (scale uncert.) } \mathrm{pb} .
$$

Together with the LO prediction of $0.91_{-0.20}^{+0.29} \mathrm{pb}$ (including scale uncertainties) this results in a moderate K-factor of about 1.34. The origin of the discrepancy between theory and experiment is presently under investigation ${ }^{1}$.

\section{References}

[1] T. Aaltonen et al. [CDF collaboration], Phys. Rev. D 79, 052008 (2009).

[2] T. Aaltonen et al. [CDF Collaboration], arXiv:0909.1505 [hep-ex].

[3] V. M. Abazov et al. [D0 Collaboration], Phys. Rev. Lett. 94, 091802 (2005); Phys. Rev. Lett. 94, 161801 (2005).

\footnotetext{
${ }^{1}$ See also talks given at the workshop Northwest Terascale Research Projects $W+b$ quark physics at the LHC, held at the University of Oregon, http://physics.uoregon.edu/ soper/TeraWWW2.
} 

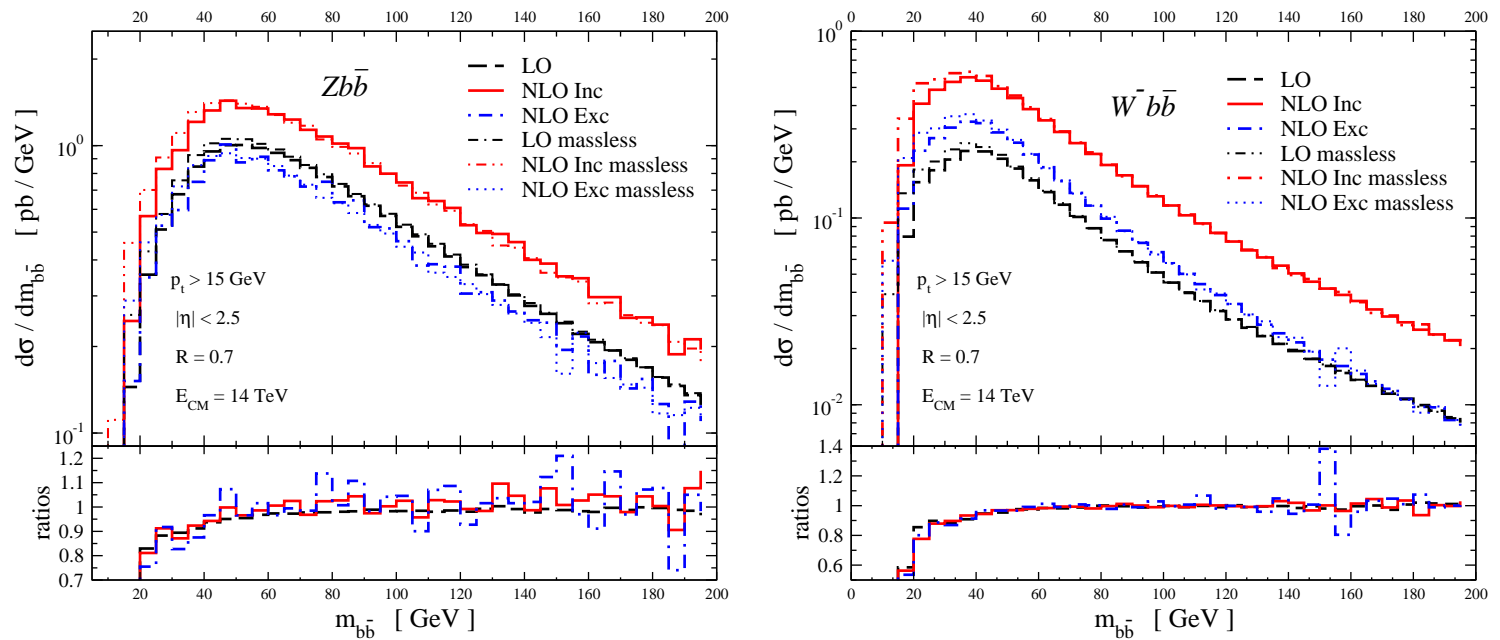

Figure 3: LO (black), NLO inclusive (red) and NLO exclusive (blue) $m_{b \bar{b}}$ distributions for Zbb (left hand side) and $W b b$ (right hand side) production at the LHC derived with $m_{b} \neq 0$ (LO: dashed, NLO inclusive: solid, NLO exclusive: dash-dotted) and from MCFM with $m_{b}=0$ (LO: double-dashed/dotted, NLO inclusive: dashed/double-dotted, NLO exclusive: dotted). The lower window shows the ratio of the distributions for massive and massless $b$ quarks, $d \sigma\left(m_{b} \neq 0\right) / d \sigma\left(m_{b}=0\right)$ (LO: dashed, NLO inclusive: solid, NLO exclusive: dash-dotted). Taken from Ref. [10].

[4] J. M. Campbell, R. K. Ellis, F. Maltoni and S. Willenbrock, Phys. Rev. D 69, 074021 (2004); Phys. Rev. D 73, 054007 (2006); Phys. Rev. D 75, 054015 (2007).

[5] Z. Bern, L. J. Dixon and D. A. Kosower, Nucl. Phys. B 513, 3 (1998); Z. Bern, L. J. Dixon, D. A. Kosower and S. Weinzierl, Nucl. Phys. B 489, 3 (1997); R. K. Ellis and S. Veseli, Phys. Rev. D 60, 011501 (1999); J. M. Campbell and R. K. Ellis, Phys. Rev. D 62, 114012 (2000); J. M. Campbell and R. K. Ellis, Phys. Rev. D 65, 113007 (2002).

[6] J. M. Campbell, R. K. Ellis and D. L. Rainwater, Phys. Rev. D 68, 094021 (2003).

[7] F. Febres Cordero, L. Reina and D. Wackeroth, Phys. Rev. D 74, 034007 (2006).

[8] F. Febres Cordero, L. Reina and D. Wackeroth, Phys. Rev. D 78, 074014 (2008).

[9] F. Febres Cordero, arXiv:0809.3829 [hep-ph].

[10] F. Febres Cordero, L. Reina and D. Wackeroth, Phys. Rev. D 80, 034015 (2009).

[11] J. M. Campbell et al., arXiv:hep-ph/0405302; K. A. Assamagan et al. [Higgs Working Group Collaboration], arXiv:hep-ph/0406152; M. Kramer, Nucl. Phys. Proc. Suppl. 135, 66 (2004).

[12] S. Dawson, C. B. Jackson, L. Reina and D. Wackeroth, Int. J. Mod. Phys. A 20, 3353 (2005).

[13] J. M. Campbell, R. Frederix, F. Maltoni and F. Tramontano, Phys. Rev. Lett. 102, 182003 (2009).

[14] J. M. Campbell et al., Phys. Rev. D 79, 034023 (2009).

[15] J. M. Campbell et al., in preparation.

[16] J. Campbell, R. K. Ellis, webpage: mcfm.fnal.gov.

[17] H. L. Lai et al. [CTEQ Collaboration], Eur. Phys. J. C 12, 375 (2000).

[18] J. Campbell, F. Febres Cordero, L. Reina, private communication. 